\title{
Numerical Analysis of Stable Brine Displacements for Evaluation of Density- Dependent Flow Theory
}

\author{
S. J. Watson' and D. A. Barry ${ }^{2}$ \\ 'Golder Associates Pty. Ltd., 441 Vincent Street, West Leederville WA 6007 Australia \\ ${ }^{2}$ School of Civil and Environmental Engineering and Contaminated Land Assessment and Remediation Research Centre, \\ The University of Edinburgh, Edinburgh EH9 3JN U.K.
}

Received 7 March 2000; Revised 1 September 2000; accepted 14 September 2000

\begin{abstract}
A numerical modeiling approach, used for the analysis of stable brine displacement results, is described. The purpose of the analysis is to evaluate recent theoretical advances in modelling of density-dependent flow and transport. The data analysis involved two stages. A model based on the classical density-dependent transport theory was developed for the first stage of the analysis. Transport parameters were obtained using a sequential fitting method. The numerical model was then modified to incorporate a non-linear dispersion theory that had been recently proposed for high concentration displacements. This modified model was used to reanalyse the results obtained from the brine displacement experiments. Overall, the results of the analysis indicated that a modified transport model was required to adequately describe stable brine displacements. $\mathcal{O} 2001$ Elsevier Science Ltd. All rights reserved
\end{abstract}

\section{Introduction}

Brine transport through porous media occurs in many situations, e.g., disposal of transuranic radioactive wastes in salt domes (Grambow et al., 1998). In mining, also, there are contamination sources having a high salt concentration. Examples include the highly saline waste leachate contained within tailings disposal areas in the Western Australian goldfields (Fujijasu et al., 1996) and the brine leachate generated from potash tailings and brine ponds associated with the potash, oil and gas industries in western Canada (Barbour and Yang, 1993).

Typically, numerical models describing the movement of brine (high concentration salt solutions) through porous media are based on classical transport theory. This theory takes

Correspondence to: D. A. Barry, School of Civil and Environmental Engineering and Contaminated Land Assessment and Remediation Research Centre, The University of Edinburgh, Edinburgh EH9 3JN United Kingdom. Ph. +44 (131) 650-7204, Fax. +44 (131) 650-7276, Email: A.Barry@ed.ac.uk into account, for example, the density and viscosity variations that characterise brine transport. However, in recent years, new experimental results and theoretical analyses have raised questions concerning the applicability of the classical theory to the transport of high concentration solutions, particularly the classical formulation of Fick's law. In order to provide improved theoretical predictions, Hassanizadeh and Leijnse (1988) presented extended versions of Darcy's law and Fick's law applicable to high concentration solute transport. Experimental evidence indicated that the extended formulations did not adequately describe high concentration displacements (Hassanizadeh, 1990).

Following these experimental findings, Hassanizadeh and Leijnse (1995) examined a number of modified versions of Fick's law. They presented a non-linear dispersion theory that was shown to predict both low and high concentration displacements for a unique set of parameter values. The theory was also found (Schotting et al., 1999) to fit wcll the experimental results for high concentration displacements obtained by Moser (1995). However, the fitted dispersion coefficient was found to be considerably lower than that determined by Hassanizadeh and Leijnse (1995) for a porous medium of similar median grain size. This difference is likely to have been due to different grain Péclet numbers (and flow rates) between the two sets of experiments. Nonetheless, there is still a need for further experimental data and analysis in order to evaluate fully the new theory.

Recently, we completed a series of laboratory column experiments designed to obtain, over a range of flow rates, high quality displacement results for both low and high concentration solutions. In all cases, the experiments involved unconditionally stable (i.e., upward) displacement of salt-free water by various brine solutions. The experimental results were analysed to check the applicability of the theoretical models to high concentration displacements and to examine the effect of fluid velocity on the transport parameters. Further details of the experiments are available elsewhere (Anderson et al., 1996; Anderson and Barry, 1997).

The analysis of the experimental results relies on a numer- 
ical solution of the governing water flow and brine transport equations. Thus, we developed a numerical model to simulate stable brine displacements through saturated porous media. The model included the classical formulation of Darcy's law and Fick's law; this was used for the first stage of data analysis. In a second stage, the numerical model was extended to incorporate the modified version of Fick's law presented by Hassanizadeh and Leijnse (1995), and the experimental data were reanalysed.

This paper presents first a description of the numerical model and describes the process used to evaluate the data obtained from the stable displacement experiments. In particular, the parameter estimation process used to obtain the transport parameters from each set of experimental results is described, as it is not, a priori at least, a straightforward process. In addition, some of the experimental and numerical results are also presented to illustrate the applicability of the non-linear dispersion theory to high concentration displacements.

\section{Numerical Models}

\subsection{Governing Equations}

The one-dimensional numerical model developed to analyse the high concentration displacement results is based on the classical transport theory for density-dependent solute transport (e.g., Voss, 1984). The governing equations for the basic (i.e., classical) numerical model are summarised below (a notation list is given at the end of the paper).

Conservation-of-mass equations:

$\frac{\partial(n \rho)}{\partial t}+\frac{\partial(\rho q)}{\partial x}=0$

$\frac{\partial(n \rho \omega)}{\partial t}+\frac{\partial(\rho \omega q)}{\partial x}+\frac{\partial J}{\partial x}=0$.

Conservation-of-momentum equations:

$q=-\frac{k}{\mu}\left(\frac{\partial p}{\partial x}+\rho g\right)$

$J=-\rho n D_{h} \frac{\partial \omega}{\partial x}$

Equations of state:

$\rho=\rho_{0} \exp (\gamma \omega)$

$\mu=\mu_{0}\left(1.00+1.85 \omega-4.10 \omega^{2}+44.5 \omega^{3}\right)$.

In eq. 5 , the effect of pressure changes on density have been ignored as insignificant for the experiments analysed here (e.g., Hassanizadeh and Leijnse, 1988). Also, Hassanizadeh and Leijnse (1988) give $\gamma=0.6923$. The reference states, $\rho_{0}=998.23 \mathrm{~kg} / \mathrm{m}^{3}$ and $\mu_{0}=10^{-3} \mathrm{Ns} / \mathrm{m}^{2}$, are for salt-free water as measured at $20^{\circ} \mathrm{C}$ and $1 \mathrm{~atm}$, conditions that are appropriate for our experiments.

\subsection{Model Discretisation and Formulation}

The conservation of mass equations were discretised using the Crank-Nicolson finite-difference scheme (Noye, 1982; Barry et al., 1983, 1987), subject to the conservation-of-momentum equations and the equations of state. Details of the numerical scheme are given by Anderson (1997). This scheme gives an implicit and unconditionally stable representation of the governing equations. The discretised equations are second-order accurate in time and space. The numerical solution was tested extensively to ensure that it gave accurate and reliable solutions of the governing model, as discussed below.

A set of matrix equations for each point in space can be developed from each discretised equation. The set of matrix equations are of the form $\mathbf{A p}=\mathbf{b}$ and $\mathbf{C w}=\mathbf{d}$, where $\mathbf{A}$ and $\mathbf{C}$ are tridiagonal coefficient matrices, vectors $\mathbf{p}$ and $\mathbf{w}$ contain the unknown pressure and salt mass fraction $(S M F)$ to be calculated, and vectors $\mathbf{b}$ and $\mathbf{d}$ contain the calculated solution based on $\mathbf{p}$ and $\mathbf{w}$ values calculated in the previous time step.

The basic density-dependent numerical model was written to solve the two sets of matrix equations, subject to appropriate initial and boundary conditions. The model solved the equations by fixed-point iteration and sufficient conditions were satisfied for convergence of the numerical model (e.g., Giles, 1987). The iterations therefore converged to the exact solution of the discretised classical density-dependent governing equations, at least to within the discretisation error.

The code for the numerical model was written in FOR$T R A N$. The code operated by solving iteratively the two matrix equations in space for successive time steps, starting from a known set of initial conditions, and subject to the specified boundary conditions.

This basic numerical model was used for the first stage of the analysis of the experimental data. The model was then extended to incorporate the following non-linear dispersion equation proposed by Hassanizadeh and Leijnse (1995) to describe high concentration-gradient dispersion in porous media:

$(1+\beta J) J=-\rho n D_{h} \frac{\partial \omega}{\partial x}$,

where $\beta$ is a soil-dependent coefficient with dimensions of $L^{2} T / M$.

The non-linear dispersion equation was incorporated into the basic numerical model to form a modified version of the density-dependent transport model, in which eq. 7 replaced the classical formulation of Fick's Law.

\subsection{Boundary Conditions}

The boundary conditions used in the numerical model were selected to simulate the laboratory experiments as closely as possible. Measured pressure data at the influent boundary and measured flow rate data at the effluent boundary were used for the boundary conditions applied to the fluid mass 
balance matrix equation. Measured solute concentrations at the influent and effluent boundaries were used as the boundary conditions of the solute mass balance matrix equation.

The measured pressure and concentration boundary conditions were incorporated into the matrix equations as known values at the nodal points $i=0$ and $i=N$ (corresponding to the ends of the one-dimensional flow domain) at each time step, where $N$ is the number of increments in the $x$ direction. The measured flow rate at the effluent boundary was converted to a known pressure using a backwards difference discretisation and then included as a known pressure at the nodal point $i=N$. The accuracy of the backwards difference discretisation was consistent with the accuracy of the discretised governing equations, obtained using the CrankNicolson finite-difference scheme.

\section{Model Verification}

The basic density-dependent numerical model was verified for low concentration solute transport by comparing the numerical model results with solutions of the advection-dispersion equation. The governing equations (eqs. 1 to 6 ) reduce to the advection-dispersion equation when the influent solution concentration and existing pore fluid concentrations are vanishingly small. The classical density-dependent numerical model was therefore verified for low concentration displacements by comparing the model results with the analytical solution for the advection-dispersion equation derived by Ogata and Banks (1961) for a semi-infinite column. The density-dependent numerical model was also checked against an independent numerical solution of the advectiondispersion equation that was discretised using a Crank-Nicolson finite-difference scheme. The boundary conditions used in the advection-dispersion numerical model were matched to the boundary conditions used to solve the classical densitydependent numerical model.

The problem examined in the verification simulations was a $0.1 \mathrm{~g} / \mathrm{l}$ displacement through a $0.4 \mathrm{~m}$ long soil column at a volume flux of $10^{-4} \mathrm{~m} / \mathrm{s}$. The intrinsic permeability of the soil was $10^{-10} \mathrm{~m}^{2}$, the porosity was 0.4 , and the hydrodynamic dispersion coefficient was $5 \times 10^{-7} \mathrm{~m}^{2} / \mathrm{s}$. Solute breakthrough curves were determined at $x=0.3 \mathrm{~m}$ for the density-dependent numerical model, the analytical solution of the advection-dispersion equation and the numerical model for the advection-dispersion equation. At this location the downstream boundary condition has a negligible effect on the measured concentrations (Parlange et al., 1992) so, in effect, a semi-infinite domain was being simulated.

The three solute breakthrough curves are presented in Fig. 1. The solute breakthrough curves show that the classical density-dependent numerical model agreed closely with the numerical and analytical solutions of the advection-dispersion equation. The density-dependent numerical model was therefore shown to accurately reflect the governing equations at low solute concentrations.

Attempts were made to verify the basic numerical model

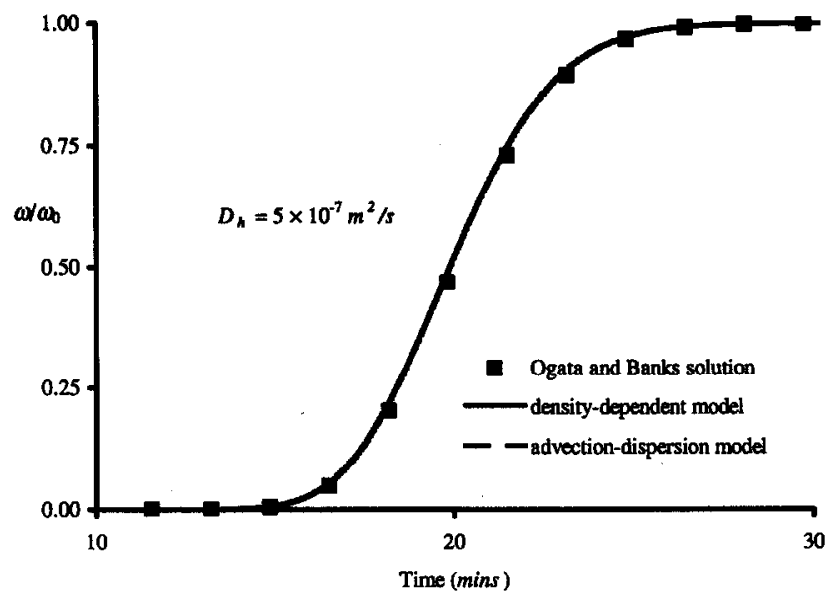

Fig. 1. Comparison of the density-dependent numerical model with a numerical and analytical (Ogata and Banks, 1961) solution of the advectiondispersion equation.

at high concentrations by comparing the results to another popular numerical model, SUTRA (Voss, 1984). SUTRA is a finite-element model that simulates saturated and unsaturated, density-dependent groundwater flow and energy transport or chemically reactive, single-species solute transport.

Numerical dispersion problems were encountered with SUTRA so it was not possible to verify the solute transport results obtained from the basic density-dependent model for high concentration displacements. Furthermore, it was also not possible to verify the shape of the pressure curves simulated by the basic density-dependent numerical model because the viscosity in $S U T R A$ is a constant.

As it was not possible to verify the classical density-dependent numerical model for high concentration displacements, the performance of the numerical model was checked for both low and high concentration displacements to ensure that the model was accurately reflecting the governing equations, and to check that the results were reliable and consistent over a range of concentrations.

In summary, a well established numerical solution method was adopted and used after considerable testing. In the following, grid-independent results were used in all cases.

\section{Experiments}

One-dimensional laboratory column experiments were conducted to obtain high quality displacement results for both low and high concentration solutions, over a range of flow rates. The column experiments were stable upward displacement experiments conducted at a constant flow rate.

The laboratory column apparatus consisted of an acrylic column with a detachable base and piston top cap. The inter.nal diameter of the column was $80 \mathrm{~mm}$, and the final packed soil length was approximately $435 \mathrm{~mm}$. The column was instrumented with pressure transducers and soil conductivity probes, allowing the pore fluid pressure and concentration to 


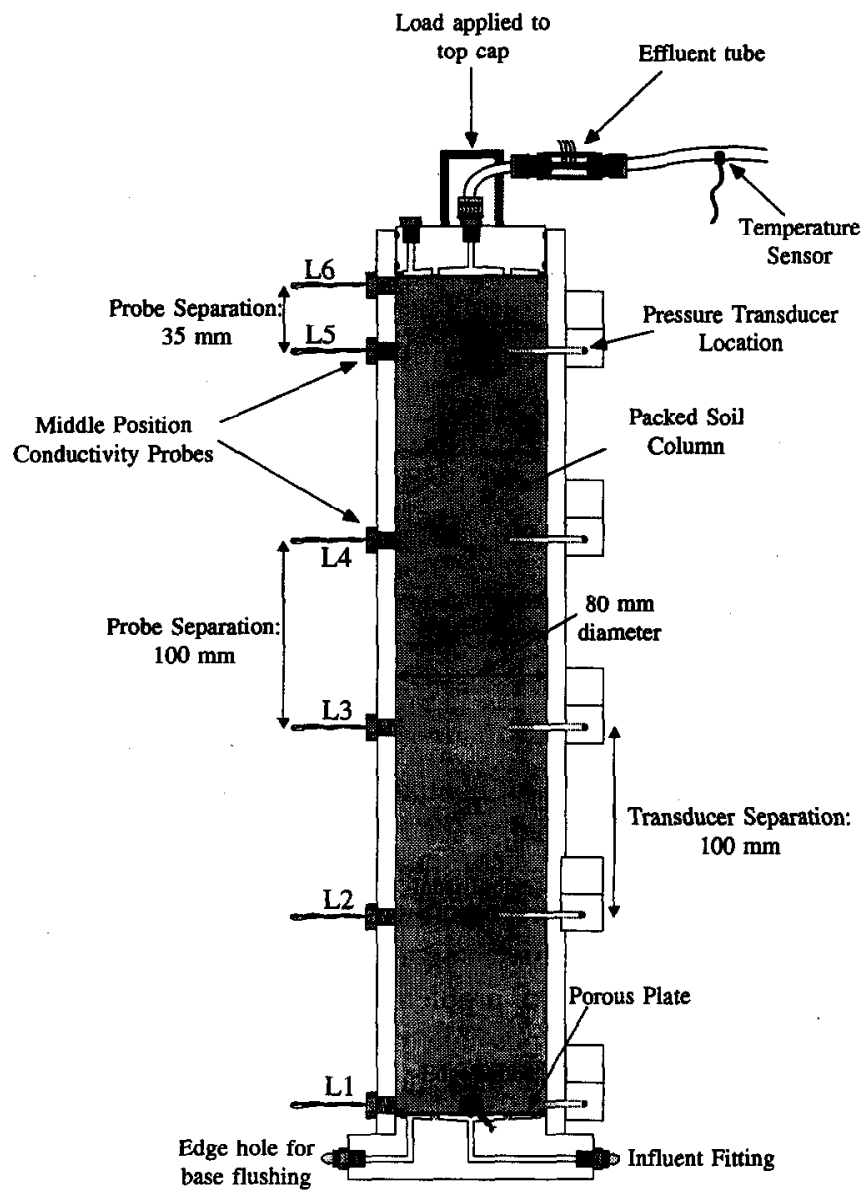

Fig. 2. Laboratory column apparatus.

be measured and logged at various locations along the length of the column, labelled $L 1$ to $L 6$. The conductivity probes were laboratory-scale 4-pin conductivity probes developed at The University of Western Australia (Watson and Culligan, 1999). A diagram of the column apparatus is given in Fig. 2.

The influent solutions used in the experiments were sodium chloride solutions with concentrations of 5 and $200 \mathrm{~g} / \mathrm{l}$. The test soil was a coarse sand which had average grain size of $0.73 \mathrm{~mm}$. The column was packed with the sand under wet conditions with vibration to give a high packed soil density.

Displacement experiments were conducted at five different flow rates with effluent flux values in the range $0.8-15.6$ $\mathrm{mm} / \mathrm{min}$. At each flow rate a low concentration $(5 \mathrm{~g} / \mathrm{l})$ and a high concentration $(200 \mathrm{~g} / \mathrm{l})$ displacement experiment was conducted, resulting in 10 displacement experiments. An additional low concentration displacement was also conducted at a low flow rate of $0.2 \mathrm{~mm} / \mathrm{min}$.

The data obtained for each experiment consisted of conductivity data from the soil probes and effluent tube probes, pore pressure data and cumulative effluent mass data. The data were then converted into solute breakthrough curves, pore pressure curves and an effluent flux curve.

\section{Parameter Estimation}

The first stage of data analysis involved the estimation of transport parameters using the classical density-dependent transport model. For the second stage, the experimental data was analysed using the modified transport model to obtain an alternate set of transport parameter estimates for each displacement experiment.

The transport parameters were estimated by fitting numerical simulation curves to the experimental curves for each displacement experiment. The curves were fitted by incorporating the numerical model, described in Section 2, into a leastsquares optimisation program, called LSQOPT. This optimisation program, which follows Marquardt (1963), minimises the sums of the squares of the residuals between the experimental measurements and numerical predictions (Parker and van Genuchten, 1984).

For the first stage of data analysis, the basic numerical model was incorporated into LSQOPT. Two versions of the least-squares optimisation program were developed. The first version was used to fit a numerical solute breakthrough curve to each experimental breakthrough curve to obtain estimates of the soil porosity and hydrodynamic dispersion coefficient for the four soil regions (regions $I$ to $I V$ ) shown in Fig. 2. The second version was used to fit a numerical pressure curve to each experimental pressure curve to obtain estimates of the soil intrinsic permeability for the four soil regions. Experimental data measured at the column entrance and exit were used as the boundary conditions for the simulations used to determine parameter values via least squares optimisation.

Prior to obtaining parameter estimates using the least squares optimisation programmes, approximate values were estimated for the soil parameters in the four soil regions. The parameters for the small soil region above region $I V$ were assumed to be equal to the soil parameters in region $I V$.

Values for the soil porosity and hydrodynamic dispersion coefficient were then estimated for region $I$ using the first version of the optimisation program to fit a numerical solute breakthrough curve to the experimental breakthrough curve measured at level $L 2$ (shown in Fig. 2). This technique was able to be used because the soil porosity and hydrodynamic dispersion coefficient for the soil region were completely determined by the shape of the breakthrough curves at either end of the soil region (levels $L 1$ and $L 2$ ), the length of the soil region, the time separation of the breakthrough curves and the flow rate through the column, all of which were measured directly.

Once the evaluated porosity and dispersion coefficient values were entered into the data input file, the intrinsic permeability of region $I$ was determined using the second version of the optimisation program to fit a numerical pressure curve to the experimental pressure curve at level $L 2$. The evaluated intrinsic permeability value was then entered into the input file. This procedure of first determining the porosity 


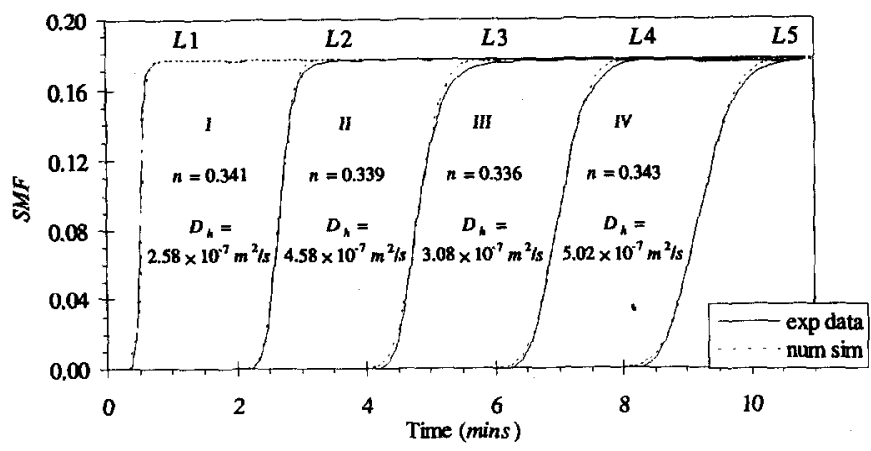

Fig. 3. Experimental (exp data) and fitted numerical (num sim) solute breakthrough curves.

and hydrodynamic dispersion coefficient of the soil region, followed by the intrinsic permeability, was then repeated for soil regions $I I$ to $I V$. The porosity was evaluated before the intrinsic permeability value because the permeability value for each soil region is directly dependent on the soil porosity.

The approximate values estimated for each soil region prior to running the optimisation simulations were initially required as input values for the optimisation programs. Once a set of values for a soil region had been estimated with the optimsation program, the approximated values were replaced by the fitted parameter values in the input file, and the process repeated until all of the approximated values had been replaced by fitted values. This sequential fitting method provided reliable values for the various flow and transport parameters characterising the density-dependent flow system.

To complete the analysis, the soil parameters determined for the four soil regions were entered into the input data file. A numerical simulation of the displacement was then conducted using the coupled density-dependent transport model and the numerical results were compared to the experimental results. A comparison of the experimental and numerical solute breakthrough curves for the $200 \mathrm{~g} / \mathrm{L}$ displacement conducted at the highest flow rate is given in Fig. 3. A comparison of the experimental and numerical pressure curves at level $L 5$ for the same displacement is given in Fig. 4. Clearly, the numerical simulations fit the measured data extremely well.

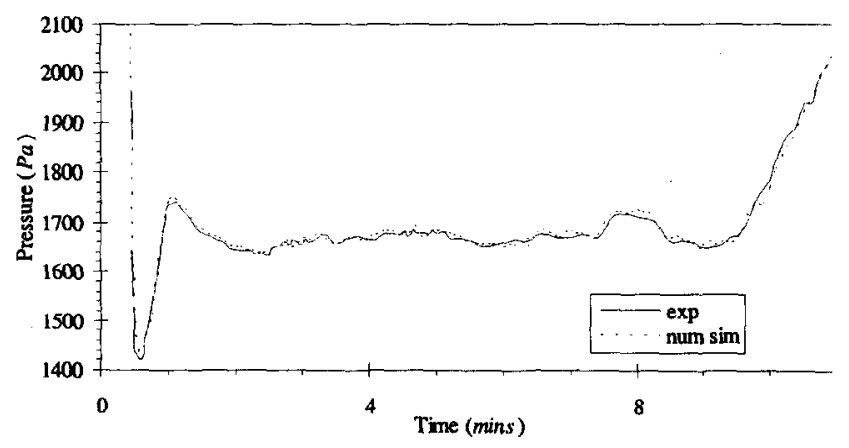

Fig. 4. Experimental (exp) and fitted numerical (num sim) pressure curves.
For the second stage of data analysis, the modified transport model was incorporated into $L S Q O P T$ to obtain estimates of $\beta$ in eq. 7. A non-linear hydrodynamic dispersion equation (shown in Fig. 5), based on results obtained during the first stage of data analysis for low concentration displacements, was also incorporated into the modified transport model. The soil porosity and intrinsic permeability values determined from the first stage of data analysis were entered as input data. The optimisation program was then used to fit the numerical solute breakthrough curve at level $L 5$ to the experimental curve at level $L 5$ measured during the high concentration displacements to obtain estimates of the transport parameter $\beta$ for each high concentration displacement.

\section{Discussion and Conclusions}

The column experiments were carefully designed to produce high quality experimental data for verification of theoretical models. In particular, the apparatus was tested to ensure that uniform solute transport fronts were maintained with minimal side-wall effects. Internal measurements of pore fluid concentration at known locations were found to provide more accurate data for model verification than measurements of effluent concentration.

The experimental data was analysed using a sequential fitting technique. This fitting method was required to obtain estimates of the soil parameters because the intrinsic permeability evaluated for a soil region is dependent on the porosity of that soil region. The two parameters can therefore not be evaluated simultaneously. Although a sequential fitting method was used, the parameters obtained were found to be self-consistent within each experiment and were also found to be consistent with results presented by other researchers.

A number of observations were made following the analysis of the experimental data and the estimation of soil parameters for each displacement. In particular, the hydrodynamic dispersion coefficient was found to be a non-linear function of the seepage velocity. This result is illustrated by Fig. 5, which shows that a nonlinear curve more accurately describes the relationship between hydrodynamic dispersion and seepage velocity than the linear curve.

The dispersion results presented in Fig. 5 also show that hydrodynamic dispersion is lower for high concentration displacements than for low concentration displacements at any particular seepage velocity. This result indicates that the classical density-dependent transport model is not adequately describing the dispersion of the solute front at low and high concentrations. The experimental results were therefore analysed using the modified formulation of Fick's law proposed by Hassanizadeh and Leijnse (1995).

The second stage of data analysis resulted in an estimate of the transport parameter $\beta$ for each high concentration displacement experiment, shown in Fig. 6. The results presented in Fig. 6 show that $\beta$ varies linearly and inversely with seepage velocity in $\log -\log$ space.

As a final check on the applicability of the modified formu- 


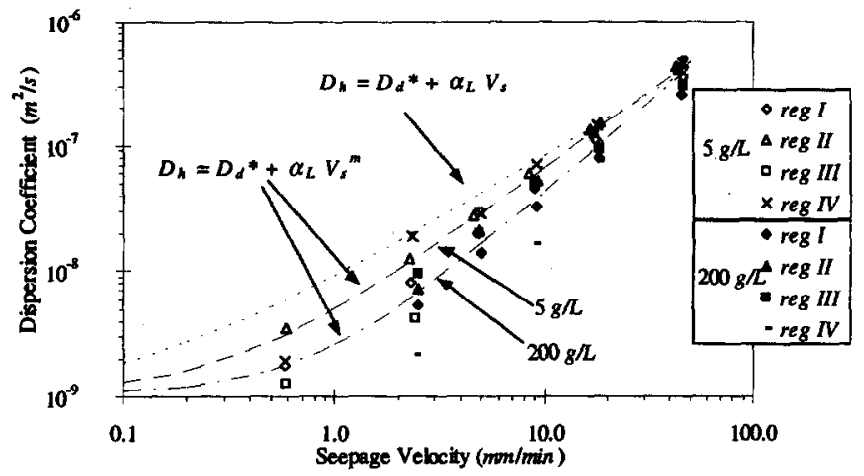

Fig. 5. Fitted hydrodynamic dispersion results for the displacement experiments. For $m=1$, the results are obtained for $\alpha_{L}=5 \times 10^{-4} \mathrm{~m}$. In the non-linear case, $\alpha_{L}=2.71 \times 10^{-3} \mathrm{~m}$ and $m=1.22$ for the $5 \mathrm{~g} / \mathrm{L}$ displacements, and $\alpha_{L}=1.18 \times 10^{-2} \mathrm{~m}$ and $m=1.44$ for the $200 \mathrm{~g} / \mathrm{L}$ displacements.

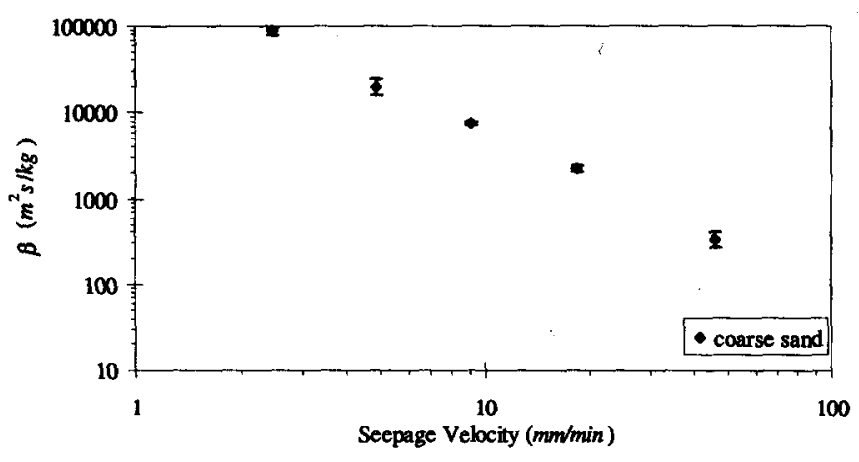

Fig. 6. $\beta$ values (see eq. 7) estimated from the high concentration displacement experiments. Bars are the estimated $95 \%$ confidence intervals. lation of Fick's law, the relationship between $\beta$ and seepage velocity was incorporated into the modified version of the numerical model. This model was found to predict accurately both low and high concentration displacements (Anderson, 1997). These results all indicate that the theory presented by Hassanizadeh and Leijnse (1995) is more accurate than classical theory for predicting stable brine displacements in porous media.

\section{Acknowledgements}

This research was conducted as part of a PhD project in the Geomechanics Group at the Department of Civil Engineering, The University of Western Australia.

\section{Notation}

\section{Bold or Italicised}

A coefficient matrix in pressure equation $\left[T^{2} / L^{2}\right]$

b forcing term in pressure equation $\left[M / L^{3}\right]$

C coefficient matrix in transport equation $\left[M / L^{3}\right]$

d forcing term in transport equation $\left[M / L^{3}\right]$

$D_{h} \quad$ hydrodynamic dispersion coefficient $\left[L^{2} / T\right]$

$g$ magnitude of gravitational acceleration $\left[L / T^{2}\right]$

$i \quad$ spatial node

$J \quad$ dispersive flux of solute $\left[M / L^{2} T\right]$

$k \quad$ permeability $\left[L^{2}\right]$

$m \quad$ power in nonlinear dispersivity relationship

$n \quad$ porosity

$N \quad$ number of increments

$p \quad$ pressure $\left[M / L T^{2}\right]$

p vector of pressures $\left[M / L T^{2}\right]$

$q \quad$ flux $[L / T]$

$S M F \quad$ Salt Mass Fraction

$t \quad$ time $[T]$

$V_{s} \quad$ seepage velocity $(=q / n)[L / T]$

w vector of salt mass fraction values

$x \quad$ position $[L]$

Greek

$\alpha_{L} \quad$ dispersivity $[L]$

$\beta \quad$ coefficient $\left[L^{2} T / M\right]$

$\gamma \quad$ coefficient

$\Delta$. discretisation of .

$\mu \quad$ viscosity $[M / L T]$

$\rho \quad$ density $\left[M / L^{3}\right]$

$\omega$ mass fraction

Other

0 (subscript) reference state

\section{References}

Anderson, S. J., An Experimental Investigation of High Concentration Displacements in Saturated Porous Media, Ph.D. thesis, Department of Civil 
Engineering, The University of Western Australia, Nedlands, Western Australia, 1997.

Anderson, S. J. and Barry, D. A., Experimental investigation of brine transport through sand, in Environmental Geotechnics: Proceedings of the 1st Australia-New Zealand Conference on Environmental GeotechnicsGeoenvimnment 97, Melbourne, Victoria, Australia, 26-28 November, edited by A. Bouazza, J. Kodikara, and R. Parker, pp. 353-358, A. A. Balkema, Rotterdam, Brookfield, 1997.

Anderson, S. J., Barry, D. A., and Culligan-Hensley, P. J., Experimental evaluation of a brine transport model, in Environmental Geotechnics: Proceedings of the Second International Congress on Environmental Geotechnics Osaka, Japan, 5 - 8 November, edited by M. Kamon, pp. 193-198, A. A. Balkema, Rotterdam, 1996.

Barbour, S. L. and Yang, N., A review of the influence of clay-brine interactions on the geotechnical properties of Ca-montmorillonitic clayey soils from western Canada, Canad. Geotech. J., 30, 920-934, 1993.

Barry, D. A., Starr, J. L., Parlange, J.-Y., and Braddock, R. D., Numerical analysis of the snow-plow effect, Soil Sci. Soc. Am. J., 47, 862-868, 1983.

Barry, D. A., Parlange, J.-Y., and Starr, J. L., Numerical analysis of the precursor effect, Soil Sci., 143, 309-317, 1987.

Fujijasu, Y., Fahey, M., and Newson, T., Effect of salinity on the drying behaviour of mine tailings, in Environmental Geotechnics: Proceedings of the Second International Congress on Environmental Geotechnics Osaka, Japan, 5 - 8 November, edited by M. Kamon, pp. 367-372, A. A. Balkema, Rotterdam, 1996.

Giles, J. R., Introduction to the Analysis of Metric Spaces, Cambridge University Press, Cambridge, 1987.

Grambow, B., Loida, A., and Smailos, E. J., Long-term stability of spent nuclear fuel waste packages in Gorleben salt repository environments, Nucl. Tech., 121, 174-188, 1998.

Hassanizadeh, S. M., Experimental study of coupled flow and mass transport: A model validation exercise, in ModelCARE 90: Calibration and Reliability in Groundwater Modelling, edited by K. Kovar, pp. 241-250,
IAHS Publ. No. 195, Washington, DC and Paris, 1990.

Hassanizadeh, S. M. and Leijnse, T., On the modelling of brine transport in porous media, Water Resour. Res., 24, 321-330, 1988.

Hassanizadeh, S. M. and Leijnse, T., A non-linear theory of highconcentration gradient dispersion in porous media, Adv. Water Resour., 18, 203-215, 1995.

Marquardt, D. W., An algorithm for least-squares estimation of nonlinear parameters, J. Soc. Ind. Appl. Math., 11, 431-441, 1963.

Moser, H., Einfluß der Salzkonzentration auf die Hydrodynamische Dispersion im Porösen Medium, Ph.D. thesis, Technische Universität Berlin Report No. 128, 1995.

Noye, J., Finite difference methods for partial differential equations, in $\mathrm{Nu}$ merical Solutions of Partial Differential Equations, edited by J. Noye, North-Holland, New York, 1982.

Ogata, A. and Banks, R. B., A solution of the differential equation of longitudinal dispersion in porous media, Tech. rep., U.S. Geological Survey Paper 411-A, Washington DC, 1961.

Parker, J. C. and van Genuchten, M. Th., Determining transport parameters from laboratory and field tracer experiments, Bulletin 84-3, Virginia Agricultural Experiment Station, Blacksburg, Virginia, 1984.

Parlange, J.-Y., Starr, J. L., van Genuchten, M. Th., Barry, D. A., and Parker, J. C., Exit condition for miscible displacement experiments in finite columns, Soil Sci., 153, 165-171, 1992.

Schotting, R. J., Moser, H., and Hassanizadeh, S. M., High-concentrationgradient dispersion in porous media: Experiments, analysis and approximations, Adv. Water Resour, 22, 665-680, 1999.

Voss, C. I., SUTRA, Saturated-Unsaturated TRAnsport. A finite-element simulation model for saturated-unsaturated, fluid-density-dependent ground-water flow with energy transport or chemically-reactive singlespecies solute transport, Tech. rep., Water-Resources Investigations Report 84-4369, Reston, Virginia, 1984.

Watson, S. J. and Culligan, P. J., The development of small-scale conductivity probes for solute transport experiments, Tech. Rep. G1455, Geoemchanics Group, Department of Civil Engineering, The University of Western Australia, Nedlands, Western Australia, 1999. 